\title{
Genetics in the epilepsies - a broadening concept
}

\section{J Helen Cross}

\section{UCL Great Ormond Street Institute of Child Health, London WC1N 1EH}

\author{
Email: h.cross@ucl.ac.uk
}

Over recent years we have made considerable progress in revealing the aetiology of particularly the more complex epilepsies. Advances in neuroimaging initially highlighted the range of structural causes, revealing changes only previously determined through post-mortem studies. This has enabled an understanding of the different malformations, and the likely stage in development a problem may have occurred. Further, they have increased specifically those who can be considered for curative resective surgery where focal brain abnormalities are seen. That aside with further enhancement to imaging and post processing techniques, when exactly an individual may be considered image negative has become unclear. Further, advances in genomic sequencing have furthered our understanding even more, particularly the relationship between many malformations and underlying defects in gene pathways.

Known genes in which defects or deletions may be associated with the epilepsies have increased exponentially with steps forward made in genomic sequencing. These have both provided an answer with regard to underlying cause for many families, and provided more accurate genetic counselling. Increasingly with a greater understanding of the pathways for which they code, strides have been taken in targeting these pathways in management of the epilepsies. In order to increase cost effectiveness, and minimise inefficiency, gene panels have been utilised, with increasing diagnostic success but these give answers with regard only to known epilepsy associated genes, and as the number about which we are aware increases so panels become more specialised ${ }^{1,2}$. This means we need to be targeted in our approach, and does not allow for phenotypic diversity. The wider the net is cast to find a genetic change (with more detailed testing) the more likely abnormalities will be found ${ }^{3}$

Guerrini et al within this issue highlight there is increased blurring of the boundary, if any, between genetic and structural aetiologies ${ }^{4}$. The question increasingly remains with enhanced imaging and review, as to whether all those currently highlighted as lesion negative on MRI, truly are. A genetic diagnosis does not exclude a disruption to brain structure. One could argue there are structural changes from a genetic change at any level - whether this can be visible with techniques available will be determined by the timing and place of the gene pathway disruption and the detail of the imaging. Further, negative gene panel testing does not exclude a genetic cause.

The relevance of this dilemma is increasingly apparent in clinical practice. By obtaining as much information as we can, whether structural or genetic, this provides answers for families, more accurate genetic counselling, and possibly predictors of prognosis. Does a genetic aetiology change post surgical outcome in focal malformations - this may depend on the pathway that is disrupted ${ }^{5}$ and requires further evaluation The more accurate a genetic diagnosis, the more likely we will have an understanding of the underlying pathophysiology, and will move toward precision medicine. As highlighted by Guerrini et al, moving toward casting a wider net through WES is more likely to be result in more efficient cost benefit in the longer term. 
1. Trump $\mathrm{N}$ McTague $\mathrm{A}$, Brittain $\mathrm{H}$ et al Improving diagnosis and broadening the phenotypes in early onset seizure and severe developmental delay disorders through gene panel analysis. J Med Genetics 2016; 53(5):310-7

2. Symonds J, Zuberi S, Stewart $\mathrm{K}$ et al Incidence and phenotypes of childhood-onset genetic epilepsies: a prospective population-based national cohort Brain 2019;142: 2303-2318

3. Berg AT, Coryell J, Saneto RP, Grinspan ZM, Alexander JJ, Kekis M, Sullivan JE, Wirrell EC, Shellhaus RA, Mytinger JR, Gaillard WD, Kossoff EH, Valencia I, Knupp KG, Wusthoff C, Keator C, Dobyns WB, Ryan N, Loddenkemper T, Chu CJ, Novotny EJ, Koh S. Early life epilepsies and the emrrging role of genetic testing JAMA Pediatrics 2017;171:863-871

4. Guerrini R, Parrini E, Esposito A, Fassio A, Conti V. Lesional and non-lesional epilepsies: a blurring genetic boundary. Eur J Paed Neurol in press

5. Sanders MWCB, Lemmens CMC, Jansen FE, Brilstra EH, Koeleman BPC, Braun KPJ; Dutch Collaborative Epilepsy Surgery Program (LWEC). Implications of genetic diagnostics in epilepsy surgery candidates: A single-center cohort study. Epilepsia Open. 2019 Nov 15;4(4):609-617. doi: 10.1002/epi4.12366. eCollection 2019 Dec

\section{Disclosures}

JHC has acted as an investigator for studies with GW Pharma, Zogenix, Vitaflo and Marinius. She has been a speaker and on advisory boards for GW Pharma, Zogenix, and Nutricia; all remuneration has been paid to her department. Her work is supported by the NIHR Biomedical Research Centre at Great Ormond Street Hospital \& University College London. 